Regards sur l'économie allemande

Bulletin économique du CIRAC

82 | 2007

Varia

\title{
Présidence allemande de l'UE : de la « racine carrée » à la quadrature du cercle
}

\section{Hans Stark}

\section{(2) OpenEdition}

\section{Journals}

Édition électronique

URL : http://journals.openedition.org/rea/626

DOI : $10.4000 /$ rea.626

ISBN : 978-2-8218-0860-7

ISSN : 1965-0787

\section{Éditeur}

CIRAC

\section{Édition imprimée}

Date de publication : 1 juillet 2007

Pagination : 5-12

ISSN : 1156-8992

\section{Référence électronique}

Hans Stark, « Présidence allemande de l'UE : de la « racine carrée » à la quadrature du cercle », Regards sur l'économie allemande [En ligne], 82 | juillet 2007, document 1, mis en ligne le 01 juillet 2009,

consulté le 21 avril 2019. URL : http://journals.openedition.org/rea/626 ; DOI : 10.4000/rea.626 


\section{Présidence allemande de 1'UE : de la "racine carrée "à la quadrature du cercle}

\section{Hans Stark}

Deux ans après l'échec du projet constitutionnel européen aux referendums français et néerlandais, le gouvernement fédéral, qui a assumé la Présidence du Conseil européen durant le premier semestre 2007, s'est attelé à relancer un Traité jugé pourtant lettre morte par un bon quart des États membres. Cette volonté de relance qui s'appuyait aussi sur le fait que 18 Etats membres avaient ratifié le Traité constitutionnel, intervenait à un moment crucial de l'histoire de l'Europe. Face à la crise dans laquelle l'UE a été plongée après le non français du 29 mai, la Commission avait en effet octroyé une pause de réflexion aux Européens dans l'espoir que celle-ci permette à la discussion de repartir sur des bases plus saines, plus dépassionnées et moins idéologisées. Or, en réalité, cette phase qui a duré plus de deux ans s'est avérée non pas une pause de réflexion, mais une pause dans la réflexion. Au printemps 2007, le débat constitutionnel était toujours bloqué au même point, c'est-à-dire au point mort. Depuis 2005, l'UE a donc traversé, à l'intérieur, une crise de régime et de confiance, alors qu'elle se voit obligée, à l'échelle internationale, d'exercer un pouvoir de régulation et de structuration sans cesse croissant, en particulier visà-vis de son voisinage au Sud et à l'Est.

Aussi ne fut-il pas étonnant de voir la Présidence allemande de l'UE afficher dès la fin de l'année 2006 un programme extrêmement ambitieux, que Berlin a souhaité réaliser en commun avec les deux Présidences suivantes de la Slovénie et du Portugal ("Ensemble, nous réussirons l'Europe »). Deux points essentiels ont tout particulièrement retenu son attention : la protection du climat et la sécurisation de l'approvisionnement énergétique, d'un côté et, de l'autre, la relance du processus institutionnel. Si le premier volet a été au cœur du Conseil européen des 8 et 9 mars derniers, le deuxième a alimenté les débats du Conseil des 21 et 22 juin.

Mais au-delà de ces deux points forts, la Présidence allemande a embrassé un champ beaucoup plus vaste, trop vaste sans doute. Elle s'est ainsi efforcée d'obtenir des résultats concrets dans le domaine de la lutte contre le terrorisme international et la criminalité transfrontalière. Affichant son intention de développer un " espace européen de sécurité et de stabilité ", elle a exprimé le désir de stabiliser les Balkans occidentaux, de renforcer la politique européenne de voisinage et d'intensifier les relations avec la Russie et les pays d'Asie centrale. Une attention toute particulière a enfin été accordée à l'intensification des relations avec le Proche-Orient. Mais les ambitions de la Présidence allemande ne s'arrêtaient pas là. Berlin souhaitait également contribuer à l'achèvement du marché intérieur et renforcer la compétitivité des entreprises européennes, s'engager en faveur d'une politique économique axée sur la croissance et la stabilité, permettre une législation plus efficace et moins coûteuse de l'UE et, enfin, promouvoir la politique européenne des transports. Vaste programme pour une Présidence limitée à 6 mois et semée d'embûches.

Néanmoins, nombreux, peut-être même trop nombreux, furent ceux qui avaient attaché des espoirs immenses à cette Présidence. Ne serait-ce qu'en raison de
Deux moments forts : énergie et processus constitutionnel

Une feuille de route très (trop ?) chargée

Des attentes immenses placées dans le pouvoir d'Angela Merkel 
l'aura qui entoure la chancelière. Femme de poigne, mais résolument pro-européenne, elle jouit de l'image d'une " Margaret Thatcher à l'envers ", c'est-à-dire de l'image d'une femme politique capable de s'imposer comme jadis la Dame de Fer, mais cette fois-ci au profit de la construction européenne. Si Angela Merkel jouit de cette image, c'est pour plusieurs raisons. On lui reconnaît tout d'abord un grand talent en matière de politique étrangère, domaine où elle excelle. Lors du sommet de Bruxelles, sous la Présidence britannique, les 15 et 16 décembre 2005, les éloges ont fusé pour le rôle joué par la chancelière à l'occasion de son premier Conseil européen où elle aurait réussi à agir comme médiateur entre les grands États membres, et entre Français et Britanniques en particulier.

C'est ainsi que l'espoir est né au sein de l'UE qu'en 2007, après les élections en France et la clarification de la situation politique en Grande-Bretagne, la Présidence européenne de l'Allemagne pourrait donner de nouvelles impulsions et développer de nouveaux concepts pour surmonter l'impasse dans laquelle se trouve I'UE depuis le printemps 2005. De plus, la chancelière forme un tandem heureux et efficace avec son ministre des Affaires étrangères, Frank-Walter Steinmeier, l'ancien bras droit de Gerhard Schröder, qui partage ses idées et concepts.

Enfin, et peut-être surtout, Angela Merkel a profité, tout au long de l'année 2006 et du premier semestre 2007, d'un certain vide autour d'elle qui n'a pu que lui être profitable. Face à une France paralysée par la fin de l'ère Chirac et les suites du non à la Constitution européenne, une Amérique de Bush empêtrée dans la guerre en Irak, un Tony Blair sur le départ et une Italie affaiblie au plan interne, l'Allemagne de Merkel semblait être le seul parmi les grands pays occidentaux à même de faire des propositions constructives suivies d'effets réels.

\section{Le sommet à mi-parcours}

Deux sommets, deux thématiques propres

Sommet de mars : énergie, climat et Stratégie de Lisbonne

Une stratégie intégrée pour l'énergie et le climat..
Durant la première phase de la Présidence allemande, le gouvernement fédéral s'est efforcé de mettre ses ambitions institutionnelles entre parenthèses en attendant la fin de la campagne présidentielle en France. La Présidence allemande a donc affiché un double bilan, marqué par les résultats des deux sommets de mars et de juin. L'agenda des deux sommets suivait la logique des sommets européens qui veut que le premier Conseil, à mi-parcours, soit consacré aux questions économiques et le deuxième, qui clôture la Présidence semestrielle, aux problèmes politiques. C'est ainsi que la chancelière, lors d'une rencontre avec la Commission le 9 janvier 2007, a clairement défini les buts de sa Présidence, en laissant toutefois entendre qu'il est impossible pour une Présidence de résoudre tous les problèmes de l'Union européenne (voir Bulletin Quotidien Europe - BQE, 10-01-2007, p. 4).

En janvier, Angela Merkel a donc clairement souligné que la nouvelle impulsion qu'elle souhaitait donner en faveur du traité constitutionnel ne devait intervenir qu'au sommet de juin, après les élections françaises. Le sommet de mars, quant à lui, devait livrer un triple message : I'UE devait se doter des bases d'une politique énergétique européenne, avec des engagements et des objectifs contraignants, et des procédures précises. Elle devait jouer un rôle moteur à l'échelle mondiale dans le domaine de la protection du climat, en vue notamment de l'expiration de l'accord de Kyoto après 2012 (et du sommet du G-8 de juin 2007, également sous présidence allemande). Enfin, au plan économique, I'UE devait progresser sur la voie des objectifs de Lisbonne et encourager les pays qui ne l'ont pas encore fait à introduire chez eux les réformes prévues (voir REA 80/07).

Ce pari multiple a été tenu. Lors du Conseil des 8 et 9 mars 2007 à Bruxelles, les 27 se sont en effet engagés sur la voie d'une réduction des émissions de gaz à effet de serre de $30 \%$ pour tous les pays industrialisés à l'horizon 2020 
par rapport à 1990 (et de $60 \%$ à $80 \%$ d'ici 2050). Ils ont surtout souscrit à l'engagement unilatéral d'une réduction de $20 \%$ des émissions de l'UE au cas où les autres pays industrialisés refuseraient de suivre la politique de l'UE. Cet engagement est contraignant et s'inscrit dans la logique des propositions de la Commission intitulées "Énergies et climat » du 10 janvier 2007. Cette décision, qui est clairement à mettre à l'actif de la Présidence allemande, constitue une victoire pour Angela Merkel qui avait fait d'une stratégie intégrée de l'énergie et du climat une priorité absolue pour la lutte contre le changement climatique (voir BQE, 10-03-2007, pp. 4-5).

C'est dans cette logique intégrée que le Conseil européen a également adopté, toujours le 9 mars, un plan d'action global dans le domaine de l'énergie, axé sur cinq domaines jugés prioritaires : la création progressive d'un marché intérieur de l'énergie dans le domaine du gaz et de l'électricité ; le renforcement de l'approvisionnement et des mécanismes de solidarité énergétique (thème cher à la Pologne et aux Pays baltes, très inquiets face au monopole énergétique de la Russie et à son exploitation politique par le Kremlin); d'où le plaidoyer des 27 en faveur d'une politique énergétique internationale, autorisant l'UE à s'exprimer "d'une seule voix " et à mener des négociations directes entre pays consommateurs d'une part et pays producteurs et de transit d'autre part. Enfin, I'UE a adopté des objectifs quantifiés ambitieux pour l'efficacité énergétique et les énergies renouvelables, visant à économiser d'ici la fin de la prochaine décennie $20 \%$ de la consommation énergétique de l'Union et à assurer une part de $20 \%$ également d'énergies renouvelables dans la consommation énergétique totale de l'UE (voir BQE, 10-03-2007, pp. 6-8)

Ces résultats correspondent parfaitement aux objectifs que le gouvernement fédéral s'était fixé dès 2006 (Gloser, 2006). Ceci ne signifie pas en revanche que leur mise en œuvre se fera sans heurts. Berlin, tout comme Paris d'ailleurs, craint ainsi que la mise en œuvre du paquet énergétique de la Commission ne s'accompagne d'un démantèlement de ses grands groupes énergétiques. Si l'Allemagne est favorable à un marché intégré de l'énergie, elle est critique à l'égard des mesures de la Commission visant à accélérer la libéralisation du marché de l'énergie. La France et l'Allemagne risquent en effet de perdre leur position de leaders actuels du marché européen de l'énergie. Cette crainte reflète avant tout les réticences des États membres de transférer leurs compétences nationales en matière d'énergie à l'Union. Enfin, autre question délicate : la volonté allemande de sortir du nucléaire. Cette décision, prise par le gouvernement précédent et confirmée, à la demande du SPD, par la grande coalition, est diamétralement opposée à la philosophie de la Commission et de la majorité des autres Etats membres. Ces derniers, contrairement à l'Allemagne, considèrent en effet que le nucléaire est incontournable en vue des objectifs de réduction des émissions de $\mathrm{CO}_{2}$ et qu'il joue ainsi un rôle crucial dans le domaine de la lutte contre le changement climatique. Rien que ces deux points montrent donc à quel point les ambitions d'Angela Merkel risquent de se heurter à des réalités politiques qui lui échappent en partie (Notz, 2007).

\section{Une activité diplomatique intense, mais couronnée de peu de succès}

Parallèlement aux enjeux climatiques et énergétiques, la présidence allemande s'est également efforcée de progresser sur un grand nombre de dossiers relevant de la politique étrangère et de sécurité commune (PESC) et des relations extérieures de l'Union. L'agenda allemand était bien rempli. Très présents sur un grand nombre de terrains, d'ailleurs souvent minés, la chancellerie et le ministère des Affaires étrangères (Auswärtiges Amt) ont ainsi tenté de lancer de nombreuses initiatives, qu'il s'agisse du règlement de la question du Kosovo, du partenariat euro-russe, de l'intensification des relations transatlantiques, de l'approfondissement et de l'élargissement de la politique européenne de voisinage
... et un plan d'action global pour l'énergie

Restent deux points épineux : la libéralisation du marché de l'énergie et le nucléaire
Délicats dossiers de la PESC et des relations extérieures de l'UE 
Semi-échec du sommet UE/Russie des 17 et 18 mai

Un dialogue difficile avec Varsovie

Une politique européenne de voisinage renforcée
(PEV) ou de la relance du processus de paix au Proche-Orient. Ces enjeux étant à l'origine de tensions qui empoisonnent les relations internationales depuis des années, voire des décennies, il ne fallait évidemment pas s'attendre à des miracles, ni s'étonner lorsque la dynamique propre à certains d'entre eux dépasse tout simplement la capacité de médiation des Allemands. Ainsi, les nombreuses visites effectuées par Angela Merkel et Frank-Walter Steinmeier au Proche-Orient dans le but de relancer les pourparlers de paix israélopalestiniens n'eurent pas le moindre impact sur la stabilité de la région, compte tenu de la détermination du Hamas de recourir à la force pour vaincre le Fatah et de prendre le contrôle de la bande de Gaza qui s'est ainsi transformé(e), au printemps 2007, en un mini-Etat terroriste aux portes d'Israël. II en est de même en ce qui concerne le statut du Kosovo, dont le règlement ne dépend pas de I'UE, mais de la volonté de la Russie et de la Chine, membres permanents du Conseil de sécurité de l'ONU, d'accepter ou non l'indépendance de cette province à majorité albanaise.

Enfin, le sommet UE/Russie qui s'est tenu à Samara, les 17 et 18 mai derniers, n'a donné aucun résultat substantiel en vue du nouvel accord de partenariat euro-russe souhaité par l'Allemagne et la majorité des Etats membres. En réalité, loin de développer un partenariat stratégique (et énergétique) entre les deux parties, qui est d'un intérêt vital pour Berlin, Moscou et Bruxelles doivent faire face à des tensions croissantes, liées en particulier à l'embargo russe sur les produits agricoles polonais et aux problèmes bilatéraux entre la Russie d'un côté et la Lituanie (interruption de l'oléoduc Druzhba) ou l'Estonie (déplacement du monument soviétique à Tallinn) de l'autre. L'hostilité russe à l'égard du projet américain de bouclier anti-missile en Pologne et en République tchèque, les critiques de l'UE à l'égard de l'état des libertés fondamentales et des droits de I'homme en Russie, posent aussi problème. Bref, loin de parvenir à ancrer la Russie peu à peu dans les structures de coopération européennes, en vue notamment d'une politique commune de l'énergie, l'Union risque de faire de son grand voisin un adversaire politique.

Or le rôle qui incombe dans ce contexte à l'Allemagne en tant que médiateur entre Moscou et ses anciens satellites centre-européens est crucial et dépasse très largement le cadre de sa présidence : elle doit faire de la question du bouclier anti-missile un enjeu pour la PESC et parvenir à faire prendre conscience à ses partenaires polonais, tchèques et baltes de l'inefficacité et du caractère contre-productif de leurs positions nationalistes envers le Kremlin (voir Fischer, 2006 et Arbatova, 2007). Reste que le dialogue avec Varsovie, dont l'attitude anti-russe est aussi virulente que sa germanophobie, s'annonce difficile pour Berlin.

Les rapports euro-russes planent aussi sur l'évolution de la politique européenne de voisinage (PEV) de l'Union et notamment sur les relations entre I'Union et les républiques ex-soviétiques sur lesquelles Moscou exerce une influence plus ou moins directe dans le cadre de la CEI. Dans cet espace eurasiatique, l'UE fait face à des dilemmes. De la sécurité de l'approvisionnement énergétique à la défense des droits de l'Homme dans des pays régis par des régimes dictatoriaux, en passant par la lutte contre le trafic des stupéfiants et le terrorisme islamiste, l'espace non-russe de la CEl expose l'Union à des problèmes qui constituent des enjeux considérables auxquels elle ne peut pas être insensible. En même temps, il s'agit d'une zone qui fait depuis des siècles partie de la sphère d'intérêts de la Russie. Moscou n'est donc guère enthousiaste à l'idée d'un partage de son leadership avec une Union très critique à son égard en ce qui concerne la question des droits de l'homme et composée de pays dont certains affichent des positions systématiquement anti-russes. Si Frank-Walter Steinmeier, qui a effectué de nombreux voyages en Asie centrale durant la présidence allemande, plaide désormais en faveur d'une politique européenne de voisinage renforcée demandant aux pays de la CEI (hors Russie) de s'aligner au cas par cas sur les déclarations, démarches et positions de 
I'UE dans le domaine de la PESC, force est de constater que la PEV renforcée se limite pour l'instant à un approfondissement de l'intégration économique et à l'adoption progressive d'accords de libre échange. C'est un nouveau pas qui est loin d'être négligeable, mais il est encore loin de relever les défis géopolitiques de cette région (voir BQE, 20-06-2007, p. 7 et Koopmann/Lequesne, 2006).

Le point le moins conflictuel sur l'agenda diplomatique de la présidence allemande concernait les relations transatlantiques. Ainsi, au début de sa présidence, Angela Merkel avait plaidé en faveur d'un "partenariat transatlantique » dans le domaine économique et commercial, aux contours, il est vrai, modestes et donc potentiellement consensuels. En effet, ce partenariat ne vise pas la mise en place d'un marché commun (alors que l'accord de partenariat euro-russe, s'il voit le jour, prévoit un accès privilégié de Moscou au marché unique) ni d'une zone de libre échange. Les questions relatives aux droits de douane et à l'ouverture des frontières aux marchandises sont donc exclues de ce partenariat. En revanche, le gouvernement fédéral avait souhaité se concentrer sur des mesures d'harmonisation afin de surmonter tous les obstacles artificiels des échanges. Le partenariat proposé par la présidence allemande devait donc porter en particulier sur la coopération réglementaire, les marchés des capitaux, l'énergie et la protection de la propriété intellectuelle (voir BQE, 12-01-2007, p. 3), l'objectif étant de rendre les échanges plus fluides. Cette initiative de la chancelière allemande a porté ses fruits à l'occasion du sommet annuel UE/Etats-Unis qui a eu lieu à Washington le 30 avril 2007 et qui a vu l'adoption, par les deux parties, d'un «cadre » ayant pour objectif de renforcer la coopération économique transatlantique et de faciliter les procédures réglementaires afin d'approfondir l'intégration économique entre l'Union européenne et les Etats-Unis.

\section{L'apothéose polono-bruxelloise : vers un nouveau traité pour l'UE}

L'agenda de la présidence allemande n'était pas seulement chargé, il était aussi ambitieux. L'objectif principal était bien entendu la relance institutionnelle via la réanimation du traité constitutionnel de 2003, une mission jugée presque impossible par presque tous les observateurs. In fine, Angela Merkel a eu gain de cause, le Conseil européen s'étant mis d'accord, dans la nuit du 22 au 23 juin, sur le mandat d'une Conférence intergouvernementale chargée de finaliser et d'adopter avant la fin de l'année 2007 un "traité de réforme ». Mais le chemin fut long et tumultueux avec, en prime, un résultat plutôt en deçà des attentes allemandes.

Début janvier, le gouvernement allemand avait en effet annoncé son intention de présenter des propositions qui sauvegarderaient la substance du projet de Traité constitutionnel. Cette relative fermeté a encore été soulignée par la réunion, le 26 janvier à Madrid, des 18 pays qui ont ratifié la Constitution européenne. Une semaine auparavant, devant le Parlement européen, Angela Merkel avait fait part de son intention d'établir une feuille de route et de relancer les négociations sur la base non pas du traité de Nice (comme l'avait exigé Varsovie), mais du Traité constitutionnel. Elle avait également fait savoir qu'elle n'excluait pas des changements, même si elle souhaitait sauvegarder la substance du TCE. Toutefois, l'objectif principal était d'obtenir un nouveau traité au plus tard sous la présidence française de 2008 et d'achever la procédure de ratification avant les élections européennes de 2009. Or, il s'agissait là d'une mission presque impossible compte tenu des divisions au sein de l'UE sur le projet constitutionnel et, en réalité, sur la finalité politique de l'Union.

La présidence allemande a dû faire face à trois groupes de pays, aux intérêts fort divergents : le groupe des 18 qui ont ratifié le traité (certains par referendum) et qui estimaient que leurs voix devaient aussi être prises en considération ; les pays eurosceptiques, qui estimaient que le projet constitutionnel était
Des avancées sur les relations transatlantiques

Sauvegarder la substance du Traité constitutionnel

Intérêts divergents de trois groupes d'Etats 
Déclaration de Berlin : reflet des divergences, mais feuille de route possible

Vers un Traité simplifié et une CIG chargée de le négocier

Positions tranchées des Etats à la veille du sommet de juin

Pondération des voix : l'opposition de la Pologne mort et enterré (essentiellement le Royaume-Uni, la Pologne, les Pays-Bas et la République tchèque) ; enfin, la France, pays «moteur » de l'Europe, mais « responsable » de la crise institutionnelle et dont il fallait attendre l'issue des élections présidentielles pour savoir si un nouveau traité serait une fois de plus soumis à referendum (et donc rejeté) ou pas. De plus, même si l'élection de Nicolas Sarkozy marquait le retour de la France sur la scène européenne, le nouveau président n'était pas totalement en phase avec la chancelière. Favorable, à l'instar de cette dernière, à une accélération du processus moyennant la fixation, en juin, d'un calendrier contraignant et d'un mandat précis pour les deux présidences suivantes, Sarkozy s'est prononcé d'abord pour un "minitraité », puis pour un traité simplifié, alors que Merkel tablait toujours sur la sauvegarde de l'essentiel de la Constitution européenne.

Les divergences entre les 27 ont éclaté une nouvelle fois au grand jour à l'occasion de la présentation de la «Déclaration de Berlin », le 25 mars 2007, qui était censée célébrer le $50^{\circ}$ anniversaire des traités de Rome. Or, rédigé dans le plus grand secret, le texte de cette déclaration a mis en exergue tous les désaccords existants. II s'agit en effet d'un texte rédigé sur la base du plus petit dénominateur commun possible et qui écarte toutes les vraies questions de la construction européenne. Faisant référence à la dignité de l'homme et la parité des femmes, à la paix, la liberté, la pauvreté et la lutte contre le terrorisme, il n'évoque pas la Constitution, ni la PESC et fait tomber sous silence les élargissements à venir, tout comme l'espace Schengen! En revanche, recherche de compromis oblige, la Déclaration de Berlin fixe enfin une feuille de route ambitieuse (que contesteront aussitôt les responsables polonais et tchèques) en annonçant la volonté des 27 "d'asseoir l'Union européenne sur des bases communes rénovées d'ici les élections au Parlement européen de 2009 » (Déclaration de Berlin, voir www.eu2007.de).

La «Déclaration de Berlin » a donc permis de clarifier le débat dans les semaines suivantes sur deux points essentiels. Premièrement, une alliance d'Etats favorables à un «Traité simplifié » au lieu du «Traité constitutionnel » a commencé à émerger, une donnée, dont la présidence allemande devait évidemment tenir compte. Cette dernière devait donc aussi se résoudre à la disparition de symboles « quasi-constitutionnels », tels que le drapeau et l'hymne européens. Ensuite, les 27 ont approuvé la proposition allemande de convoquer une CIG le plus rapidement possible, chargée de négocier le futur traité sur la base des orientations du Conseil de juin 2007.

Ce Conseil, qui devait clore la présidence allemande, représentait donc un enjeu d'autant plus considérable pour l'avenir de l'Union que, à la veille du sommet, de nombreuses divergences subsistaient entre les 27. Ainsi, la GrandeBretagne a adopté une position maximaliste en s'opposant au projet de doter l'UE de la personnalité juridique, à l'extension du champ des décisions prises à la majorité qualifiée et à la reprise dans le nouveau Traité de la Charte des droits fondamentaux, c'est-à-dire la deuxième partie du Traité constitutionnel. Enfin, elle a refusé (tout comme Prague et La Haye) que la personne en charge de la PESC, de la PESD et des relations extérieures de l'Union porte le titre de « ministre européen des affaires étrangères ». Les Pays-Bas, quant à eux, plaidaient pour une sorte de droit de veto des Parlements nationaux.

La Pologne, très hostile au principe de la double majorité pour les votes au conseil, exigeait dans un premier temps le maintien de la pondération des voix établie à Nice, puis, depuis début juin, une méthode de calcul située à mi-chemin entre celle de Nice et celle de la Constitution, basée sur le recours à la racine carrée de la population (en millions ; selon ce système, les petits Etats auraient obtenu 2 voix, contre 6 pour la Pologne, 8 pour la France et 9 pour l'Allemagne). Varsovie a défendu sa position en arguant du fait que le système de la double majorité des votes, plus favorable aux grands qu'aux moyens Etats (ce qui est incontestable), aboutirait à une domination allemande dans I'Union. Cette domina- 
tion démographique était jugée d'autant plus insupportable par les Polonais qu'elle serait la conséquence directe de l'agression hitlérienne de 1939 à laquelle un Polonais sur cinq n'a pas survécu. D'où la demande des frères Kaczynski de comptabiliser les morts polonais dans le décompte des voix. Cette suggestion, si elle reflète l'état des relations germano-polonaises aujourd'hui, a été jugée grotesque par presque tous les États membres et s'est finalement retournée contre Varsovie.

Cette revendication a failli provoquer l'échec du sommet de Bruxelles des 21 et 22 juin 2007, dans la mesure où la question des votes n'était même pas à l'ordre du jour. Le 14 juin, la présidence allemande avait en effet adressé aux Etats membres un document de travail avec six questions ouvertes pour les négociations sur le futur Traité ; elles portaient sur la Charte des droits fondamentaux, la répartition des compétences, les symboles de l'UE, la politique étrangère, le rôle des parlements nationaux et la terminologie juridique. La question de la double majorité n'en faisait pas partie. Face à la persistance du blocage polonais à Bruxelles, Angela Merkel avait finalement envisagé, dans l'aprèsmidi du 22, de convoquer la CIG sans l'accord de Varsovie, une décision parfaitement conforme au droit européen, mais assez délicate d'un point de vue politique (la convocation d'une CIG peut être décidée à la majorité qualifiée, sans possibilité de veto ; en revanche, le traité qui résulte d'une CIG doit être signé et ratifié à l'unanimité).

Plusieurs responsables politiques, dont Sarkozy, Juncker, Blair et Zapatero, estimant qu'il fallait poursuivre les pourparlers, se sont donc lancés dans une discussion avec les deux frères Kaczynski qui fut finalement couronnée de succès. Le compromis obtenu à l'arraché entre les 26 et la Pologne rejette le calcul de la racine carrée au profit de la double majorité, mais au prix de diverses dispositions transitoires. Celles-ci prévoient le maintien des dispositions du Traité de Nice jusqu'au 31 octobre 2014, ainsi qu'une période transitoire allant du $1^{\text {er }}$ novembre 2014 au 31 mars 2017 pendant laquelle la double majorité sera appliquée, sauf si un État demande le recours au vote selon le Traité de Nice.

Par ailleurs, l'accord de Bruxelles prévoit les éléments suivants qui reflètent tous, à des degrés divers, des compromis : le futur Traité de l'UE n'aura pas de caractère constitutionnel. La Charte des droits fondamentaux ne figurera pas dans son intégralité dans le texte. Elle aura en revanche un caractère contraignant (sauf pour le Royaume-Uni). La majorité qualifiée deviendra la règle pour 51 domaines supplémentaires, dont la coopération judiciaire et policière (sauf pour Londres). Le nouveau traité instaurera une Présidence permanente et élargira les compétences du Haut Représentant de l'UE pour la PESC (qui ne porte pas le titre de ministre), comme l'avait prévu le projet constitutionnel. La procédure de codécision est étendue, tout comme le contrôle de la subsidiarité par les parlements nationaux. Enfin, le changement climatique et la sécurité énergétique figureront dans le texte (voir BQE, 24-06-2007).

Autre succès non négligeable pour Angela Merkel, le Conseil de Bruxelles a adopté un mandat très clair et précis pour la CIG convoquée pour le 23 juillet 2007. Celle-ci ne devra donc pas rouvrir la boîte de Pandore étant donné que les 27 ont déterminé le contenu et les détails du nouveau Traité. Celui-ci pourra donc être signé à la fin de l'année 2007 et ratifié au cours de l'année 2008 (sauf en cas de referendums dans certains États membres). II n'est donc pas exclu que l'UE dispose d'un nouveau traité avant les élections européennes de 2009, comme l'avait souhaité Angela Merkel.

LA PHASE DE REFLEXION, QUI FUT EN REALITE UNE LONGUE PHASE DE CRISE, prendra donc fin grâce à l'impulsion de la présidence allemande. Toutefois, les divergences qui se sont manifestées tout au long de l'année 2007 ne disparaîtront pas comme par enchantement mais s'incrusteront sous une forme ou une autre dans le nouveau traité. Accompagné de clauses d'opting-out, de
Délicate élaboration d'un compromis : ...

... sur la question des voix, le Traité de Nice s'appliquera jusqu'en 2014

Le futur Traité de I'UE ne sera pas une Constitution

En juillet 2007, une CIG donnera corps au Traité 
réserves, d'annexes, de déclarations et protocoles joints, de notes de bas de page et de renvois aux textes antérieurs, le nouveau Traité ne sera pas simplifié, comme l'avait souhaité le président Sarkozy, mais au contraire lourd, compliqué et parfaitement incompréhensible pour les citoyens de l'Union qui n'ont pas fait d'études de droit européen. Or, ce fait n'est pas de la responsabilité de la présidence allemande mais résulte de la volonté de parvenir à la quadrature du cercle : réunir l'Europe autour d'un traité unique sur fond d'absence de consensus sur la finalité de l'Europe.

\section{Indications bibliographiques :}

Arbatova N., "Russie-UE 2007 : le débat russe », Russie.Nei.Visions, n²0, juin 2007

BOURGEOIS I., "Présidence allemande de l'UE : convaincre et rassembler », Regards sur l'économie allemande, $n^{\circ} 80$, mars 2007

Bulletin Quotidien Europe, Édition spéciale Conseil européen de Bruxelles, n9453, 24 juin 2007

Bulletin Quotidien Europe, n`9342, 12 janvier 2007 (p.3)

"Conseil européen / Climat », Bulletin Quotidien Europe, n9383, 10 mars 2007 (pp. 4-5)

«Conseil européen / Énergie », Bulletin Quotidien Europe, n`9383, 10 mars 2007 (pp. 6-8)

"Ensemble nous réussirons l'Europe ", Programme de la Présidence $1^{\mathrm{er}}$ janier-30 juin 2007 (www.eu2007.de)

FISCHER S., " Die EU und Russland ", SWP-Studie S 34, décembre 2006

GLOSER G., «Energiepolitik für Europa - ein Schwerpunkt der deutschen Ratspäsidentschaft », Integration, $\mathrm{n}^{\circ} 4,2006$ (pp. 315-317)

KoOPMANn M., LeQueSNe C., Partner oder Beitrittskandidaten ? Die Nachbarschaftspolitik der EU auf dem Prüfstand, Nomos, Baden-Baden, 2006

"La Commission et l'Allemagne se penchent sur leur coopération du premier semestre 2007 », Bulletin Quotidien Europe, n`9340, 10 janvier 2007 (p. 4)

Notz K., «La politique énergétique allemande : entre impératifs nationaux et exigences communautaires ", Note du Cerfa $\mathrm{n}^{\circ} 42$, mars 2007

«UE/PEV », Bulletin Quotidien Europe, n॰9449, 20 juin 2007 (p.7)

www.eu2007.de/fr/About_the_EU/Constitutional_Treaty/BerlinerErklaerung.html 